\title{
Application of Anti-Stigma Design Heuristics for Usability Inspection
}

\author{
Abdul-Fatawu ABDULAI ${ }^{\mathrm{a}, 1}$, A Fuchsia HOWARD ${ }^{\mathrm{a}}$, Heather NOGA $^{\mathrm{c}}$, \\ Paul J YONG ${ }^{\mathrm{b}, \mathrm{c}}$ and Leanne M CURRIE ${ }^{\mathrm{a}}$

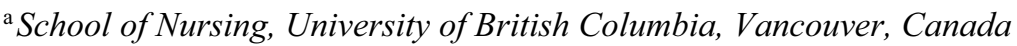 \\ ${ }^{\mathrm{b}}$ Department of Obstetrics and Gynecology, University of British Columbia, \\ Vancouver, Canada \\ 'British Columbia Women's Hospital, Vancouver, Canada
}

\begin{abstract}
User interface evaluation has become important in developing usable health care technologies. Although usability engineering methods have been applied in the design and evaluation of health care software, available heuristics focus on task-work aspects and do not address stigma associated with many health conditions. We used a previous set of heuristics and propose a new set of anti-stigma heuristics to evaluate stigmatization in health care websites. The extended set of heuristics were concurrently applied in a heuristic evaluation and a cognitive walkthrough to evaluate an endometriosis and sexual pain website. The walkthrough involved 5 tasks that required 21 actions to execute. Twenty-six usability problems were identified and recommendations for re-design were made to the design team before end-user testing. The anti-stigma heuristics received worse ratings than the traditional heuristics, resulting in several design changes that might otherwise have been missed. Thus, the new anti-stigma heuristics were a valuable contribution.
\end{abstract}

Keywords. Cognitive walkthrough, heuristic evaluation, anti-stigma heuristics, endometriosis, sexual pain, user interface, nursing informatics

\section{Introduction}

Heuristic evaluation and cognitive walkthrough are discount usability inspection methods that can improve software usability [1]. Shneiderman's 8 golden rules and Nielsen's 10 usability heuristics are often applied in the design and evaluation of health care software [2,3]. While these rules are generally applicable for an effective humancomputer dialogue, they do not address elements of stigma associated with many health conditions. Instead, they focus on task-work aspects of user interfaces from the perspective of the content, structure, and interface features of health care software, neglecting the emotional aspects of design [4]. Yet the emotional aspect of design could be more critical to a product's success than its practical features [5]. Although the current set of heuristics are still applicable in designing health care software, they fall short of necessary guidelines for anti-stigma and empowering features of health care websites. This is of particular concern for websites that intend to address sensitive topics such as sexual health and health conditions. Further, stigma has been identified as a barrier to

${ }^{1}$ Corresponding Author, Abdul-Fatawu Abdulai, School of Nursing, University of British Columbia, T201-2211 Westbrook Mall, Vancouver, British Columbia, Canada; E-mail: Fatawu@student.ubc.ca. 
uptake of sexual health interventions [6] and there is evidence that some sexual health websites can exacerbate stigma, even when their main goal is to reduce stigma [7].

In this study, we build on Nielsen's heuristics to propose a new set of anti-stigma heuristics for health care websites. The extended set of heuristics were applied in a heuristic evaluation and a concurrent cognitive walkthrough of an educational website about endometriosis and sexual pain launched in February 2021 [8]. The work reported here was designed to provide feedback to the system development team to address urgent usability problems before end-user testing and subsequent launch of the website.

\section{Methods}

The study was conducted at a large urban healthcare setting in Western Canada from September - November 2019. In this study, we integrated cognitive walkthrough and heuristic evaluation methods. To address elements of stigma associated with sexual health using web design, we extended Nielsen 10 usability guidelines by proposing 7 additional anti-stigma heuristics that, when incorporated into web design and evaluation, have the possibility to address stigma associated with sexual health. The 7 anti-stigma heuristics were identified from a literature review and were comprised of interfaceapplicable guidelines adapted from the Product Interventions Model for Stigma (PIMS model), Product Appraisal Model for Stigma (PAMS model), and other anti- stigmarelated literature [9-11]. The anti-stigma heuristics are directed at web interfaces for alleviating the effects of product-related stigma ( 2 heuristics), re-shaping the societal meaning of stigma ( 2 heuristics), and empowering people affected by endometriosis and sexual pain to effectively manage stigma (3 heuristics). The first step was identification of potential website users, their goals, background and understanding of technology. This was followed by a selection of task scenarios to guide the evaluation process. The research team developed five task scenarios that represented different ways of searching for information, including: 1. Find the psychological ways of treating sexual pain; 2. Search for information on fertility; 3 . Locate the anticipation of pain cycle; 4 . Find the role of nervous system sensitization in painful sex; and 5. Find the meaning of cul-desac. Each task scenario was broken down into a series steps needed to reach the goal.

\subsection{Evaluation Process}

Five analysts, two with Human-Computer Interaction and three with health care + web design backgrounds applied the task scenarios to concurrently conduct a cognitive walkthrough and heuristic evaluation by sequentially executing website tasks while noting instances of usability problems that a user might encounter. Analysts assessed the appropriateness of web features by asking the following: will the user be able to understand that this step is needed to get what she wants from the website (e.g., clicking on a button)?; will the user see the correct icons, search buttons they need to click or search in order to reach their goal?; will the user be able to recognize (on a subsequent visit) that this is the action they need to take?; will the user be able to understand the logic of how information is presented?. Usability problems that resulted from each question were noted in a problem reporting form. Each analyst independently reviewed the website and using our extended heuristics, they assigned severity ratings to the heuristics violated on a scale of 0 to 4 (where $0=$ no usability problem, $1=$ cosmetic usability problem, 2 = minor usability problem, $3=$ major usability problem, and $4=$ 
catastrophic usability problem). The analysts reviewed their ratings and disagreements were resolved by discussion. The mean severity ratings were calculated for each usability heuristic.

\section{Results}

\subsection{Task Analysis}

The cognitive walkthrough involved a total of 21 actions for the five tasks required to retrieve the relevant information. The maximum time for task execution was 5 minutes and the entire evaluation process lasted approximately one hour for each analyst.

\subsection{Navigation Issues Identified in the Cognitive Walkthrough}

Usability problems identified from the cognitive walkthrough process were primarily related to the absence of functions (eg: no search bar, no help buttons), absence of system's instructions (eg: no links to related pages), the ambiguity of information and tabs, and inconsistencies between the user's goal and the actions needed to achieve those goals. For example, Task 3 involved searching for information about the anticipation of pain cycle related to a sexual encounter. Analysts expected that this information would be contained in the section about pain types in order to reach their goal, but the information was contained in the section named "mechanisms" which is information about how the pain cycle processes are thought to manifest. For tasks whose execution lay within the tab "mechanisms", analysts had a hard time locating such information without first going through many pages. Some links do not lead to where expected (eg: link to "pain felt deep in the vagina" was under the symptoms tab rather than in the section about pain types). Actions and sub-actions for some tasks did not lead analysts to their goal. Clicking on symptoms and pain types tabs below the homepage, as well as the link to learn more about psychological aspects of endometriosis lead to a 404 page. Overall, there was an overlap of content that may be confusing to end-users.

\subsection{Usability Problems Identified Via Heuristic Evaluation}

A total of 26 usability problems were identified using the extended heuristic evaluation tool (see Table 1). Mean ratings ranged from 0 to 2.60. Heuristics including Error prevention, Help users recognize, diagnose, and recover from errors, and one anti-stigma heuristics (Pleasant/unoffending content) received no negative scores. Of the anti-stigma heuristics, integrating additional benefit was the heuristic most in need of refinement (Mean rating 2.6). Analysts suggested integrating chatrooms to get messages from real people, including photos of male partners, make fonts and images customizable to enhance the individual identity and self-image, and using terms that are familiar to the general public. Some analysts felt the image in the symptoms section felt too cheerful for the content. Analysts wanted to see an explanation of endometriosis on the homepage, a glossary explaining technical terms, a banner displaying sub-headings for tabs that contain more information to minimize scrolling, use bullet points rather than paragraphs, links should open in a new tab and the meaning of terms on diagrams should automatically pop-up when hovering with a mouse rather than having to click. The 
analysts also identified that some important content was left out. For example, there was not enough content on symptoms except sexual pain, and recommend to include links to sites stated under resources. For easy navigation, clicking on in-text citations should take a user to the specific reference in the reference list.

Table 1. Mean severity scores' and sample comments for the extended Nielsen-anti-stigma heuristics.

\begin{tabular}{|c|c|c|}
\hline Usability Guideline & Mean (SD) & Analysts comments \\
\hline \multicolumn{3}{|l|}{ ANTI-STIGMA HEURISTICS } \\
\hline Pleasant/unoffending content & 0 & No comment \\
\hline Unoffending images & $1.0(1.41)$ & $\begin{array}{l}\text { Only female images infer homosexuality. } \\
\text { Include male partners, transgender men }\end{array}$ \\
\hline Maximize the number of users & $2.0(1.41)$ & Information not fun enough for others \\
\hline Design to support inclusion into a society & $1.4(1.34)$ & $\begin{array}{l}\text { Technical words may limit use to only } \\
\text { people with the condition }\end{array}$ \\
\hline Strengthen the individual identity & $1.2(1.79)$ & Make fonts and images customizable \\
\hline Strengthen group identity & $0.4(0.55)$ & Clear colour branding to fit clinic and endo \\
\hline Integrate additional benefits & $2.6(1.52)$ & $\begin{array}{l}\text { Would be good to have real stories from } \\
\text { people with endometriosis }\end{array}$ \\
\hline \multicolumn{3}{|l|}{ NIELSEN'S HEURISTICS } \\
\hline Visibility of system status & $1.8(0.45)$ & Pages loads slowly \\
\hline Match between system and the real world & $1.2(0.84)$ & Technical terms. Mechanisms tabs not clear \\
\hline User control and freedom & $0.6(1.34)$ & Some links lead to other pages \\
\hline Consistency and standards & $0.6(0.89)$ & $\begin{array}{l}\text { Mechanisms could mean several things, } \\
\text { The symptom tab may be inconsistent. }\end{array}$ \\
\hline Error prevention & 0 & No comment \\
\hline Recognition rather than recall & $1.2(1.1)$ & $\begin{array}{l}\text { Difficult to know where to go first for } \\
\text { information, too much embedded information } \\
\text { in tabs, can affect recall }\end{array}$ \\
\hline Flexibility and efficiency of use & $1.4(1.14)$ & $\begin{array}{l}\text { Labels on the image don't indicate clickability, } \\
\text { use more links for easy navigation }\end{array}$ \\
\hline Aesthetic and minimalist design & $1.0(1.22)$ & $\begin{array}{l}\text { Too much content in treatment options and } \\
\text { mechanisms tabs. The visual layout should be } \\
\text { redesigned for simplicity. Need bullet points }\end{array}$ \\
\hline $\begin{array}{l}\text { Help users recognize, diagnose, and } \\
\text { recover from errors }\end{array}$ & 0 & No comment \\
\hline Help and documentation & $2(1.87)$ & No search bar, no external help buttons \\
\hline
\end{tabular}

*Rating Scores from 0-4. Low scores indicate better usability. Anti-stigma heuristics in italics.

\section{Discussion}

In this work, we employed an integrated approach that ensured identification of usability problems through an analysis of the cognitive processes required to complete a task, while allowing for an analysis and rating of violated heuristics [12]. The task scenarios were limited to searching for information, which currently constitutes the main function of the website. Most problems were minor usability problems, and only two (absence of a search bar and unclear meaning of the mechanisms tab) were considered a major usability problems. Despite identifying largely minor usability problems, which may not necessarily interfere with the system's functionality, their resolution nonetheless is necessary to reducing users' cognitive load and improve overall user experience. This work is the first of its kind to propose and apply anti-stigma heuristics for an expert evaluation of a health care website designed for patient use. Overall, the anti-stigma heuristics received more negative ratings than the Nielsen heuristics. This disparity could 
have occurred because the website was not developed based on stigma reduction principles but rather based on the principles of web design in agile development methodology [13]. Although the anti-stigma heuristics received negative ratings, analysts did not consider the website stigmatizing. In other words, analysts intimated that there was no content that could "put off" potential users.

The relatively high anti-stigma ratings suggest that anti-stigma design principles could inform the design of sexual health-related technologies for stigmatized conditions. Anti-stigma heuristics may not only help to design destigmatizing websites but could also empower users to overcome stigma and improve the overall user experience of websites for stigma-related health conditions. Analysts revealed that the website could address stigma by strengthening an individual's identity, suggesting that a person's identity could be strengthened via web technologies. However, some users may not feel comfortable opening websites on stigmatized conditions, particularly when in public places [14]. The current work was limited by only being applied to one website. Future work to explore the emotional aspects of stigma and anti-stigma from the patient perspective will help inform this program of research.

\section{Conclusion}

This usability inspection approach identified usability problems valuable to improving the usability and overall user experience. The inclusion of anti-stigma heuristics has the potential to advance patient-centered web design for improved end-user experiences.

\section{References}

[1] Kushniruk AW, Patel VL. Cognitive and usability engineering methods for the evaluation of clinical information systems. Journal of Biomedical Informatics. 2004.

[2] Cho $\mathrm{H}$ et al. A mobile health intervention for HIV prevention among racially and ethnically diverse young men: Usability evaluation. JMIR mHealth uHealth, 2018.

[3] Allen M, Currie LM, Bakken S, Patel VL, Cimino JJ. Heuristic evaluation of paper-based Web pages: A simplified inspection usability methodology. J. Biomed. Inform., 2006.

[4] Baker K, Greenberg S, Gutwin C. Empirical development of a heuristic evaluation methodology for shared workspace groupware. In Proceedings of the ACM Conference on Computer Supported Cooperative Work, 2002.

[5] Norman DA. Emotional design. Ubiquity, 2004.

[6] Weijts W, Houtkoop H, Mullen P. Talking delicacy: Speaking about sexuality during gynaecological consultations. Social Health Illn. 1993.

[7] Bauermeister JA et al. HIV and sexuality stigma reduction through engagement in online forums: Results from the HealthMPowerment Intervention. AIDS Behaviour. 2019.

[8] https://endopain.endometriosis.org

[9] Vaes K. Design for empowerment, the stigma-free design toolkit. In Advances in Intelligent Systems and Computing, 2019.

[10] MacLean R. Resources to address stigma related to sexuality, substance use and sexually transmitted and blood-borne infections. Canada Commun. Dis. Rep., 2018.

[11] Goffman E. Stigma: Notes on the management of spoiled identity. Jason Aronson, New York, N.Y., 1974.

[12] Kushniruk AW, Monkman H, Tuden D, Bellwood P, Borycki EM. Integrating heuristic evaluation with cognitive walkthrough: Development of a hybrid usability inspection method. In Studies in Health Technology and Informatics, 2015.

[13] Abrahamsson P, Salo O, Ronkainen J, Warsta J. Agile software development methods: Review and analysis. VTT Publications. 2002.

[14] Redston S, de Botte s, Smith C. Resolving embarrassing medical conditions with online health information. Int. J. Med. Inform., 2018. 